\title{
Hybrid Model Approach for Wireless Sensor Networks Coverage Improvement
}

\author{
Adda BOUALEM \\ Ibn Khaldoun University, Algeria, \\ Email: adda.boualem@univ-tiaret.dz
}

\section{Cyril DE RUNZ}

FAT, University of Tours, Blois, France

Email: cyril.derunz@univ-tours.fr

\begin{abstract}
The area coverage is a non-trivial problem in Wireless Sensors Network (WSN) due to the lack of knowledge in the minimum sensor nodes set that can cover an area of interest and limitation of energy reserve, monitoring and communication ranges. Coverage protocols address two fundamental questions: how can the coverage performance of deployed sensor nodes in a monitoring region be evaluated; and how can the coverage performance be improved when wireless sensor network cannot effectively satisfy quality of service requirements? Constructing sets of minimal nodes to optimize the coverage problem push researchers to use different techniques from different domains, such as Voronoï Diagram, Connected Dominating Set, Clustering, and others. Each technique deals with the problem by its own philosophy. Our approach aims to use a hybrid model combining Diagram of Voronoï, Clustering, and Connected Dominating Set in order to benefit from the advantages of these three models to optimize area coverage, keep connectivity, and minimize energy consumption. The simulation showed the ability of our approach to ensure optimal coverage with minimal power consumption for a longtime.
\end{abstract}

Index Terms-Wireless Sensor Networks; Clustering; Voronoi Diagram; Area Coverage; Connected Dominating Sets.

\section{INTRODUCTION}

$\mathbf{I}$ $\mathrm{N}$ the last decade, the exponential progress of wireless communication and embedded micro-sensing (MEMS) in technologies stimulate tiny and cheap wireless nodes. The Wireless Sensor Networks (WSNs) use innovative applications to enable sensing in hostile or difficult access areas such as environmental monitoring, rescue operations, pollution detection, battlefield surveillance, Health and home entertainment [13]. Moreover, each node is able to collect, to store, to process environmental information, and to communicate with neighborhood nodes. In general, WSNs could involve hundreds or even thousands of sensing nodes. It is suitable to make these nodes as energy-efficient as possible and to rely on their large numbers in order to obtain high quality of service (QoS) results. Since sensors may be spread in a random manner; a fundamental problem in a wireless sensor network is to ensure coverage and connectivity with minimal energy consumption.

978-1-7281-9015-0/20/\$31.00 (c) 2020 IEEE

\author{
Marwane AYAIDA \\ Universite de Reims Champagne Ardenne, \\ CReSTIC EA 3804, 51097 Reims, France \\ Email: marwane.ayaida@univ-reims.fr
}

Coverage is generally interpreted as the ability of a sensor network to monitor an area of interest. It can be considered as a measure of quality of service (QoS). Getting a better QoS is solving the coverage problem.

Coverage is usually interpreted as how well a sensor network will monitor a field of interest. That is to say; how to monitor a zone or an area with minimal sets of sensor nodes? This step requires an equitable deployment with good dispersion. Moreover, it impacts directly on connectivity, quality of service and lifetime of the sensor network.

The deployment is a step of dropping the nodes of sensors on the area of interest (AoI) in equitable way with instruments such as aircraft or robots, to intend a complete coverage of the geographic area. Obviously, the area coverage can be guaranteed if the deployed sensor network can retrieve the information at every point in the region. This step directly influences the coverage, the connectivity, the topology, the lifetime of the network, so the quality of service of applications to fulfill. Hence, the process of deployment and redeployment become a major necessity.

In recent years, a several researches were carried out to solve the coverage problem in wireless sensor networks based on mathematical methods (geometry as circles, triangles The grids and rhombuses), on the graph theory (as the Voronoi Diagram and the Deloney Diagram) and on the different methods of operational research domain (as scheduling techniques). The Voronoï Diagram and Delaunay Triangulation are used to solve the energetic problems, but they are not used to solve the K-coverage problems. The Clustering and Connected Dominating Sets (CDSs) are two basic techniques used to generate the backbone for wireless sensor and ad hoc networks. The clustering process divides the nodes of a network into sets of groups of nodes named clusters. In each cluster, there is a Cluster-Head (CH), which is the responsible of the coordination and the communication data between nodes in the cluster. The selection of Clusters-Head is done via a centralized strategy (by the base station) according to a certain protocols or via a localized strategy (the selection consists of a cooperation between the neighboring nodes to select the best $\mathrm{CH}$ ) according to others. Besides these protocols as (localized and centralized protocols) use geometric methods such as Voronoï diagram or Delaunay triangulation to detect holes in the network and to create an optimal topology of 
the sensors to eliminate the holes sensed. However, most of these methods do not consider in one hand the reality of the environment in which the sensor network is deployed, and do not take into consideration the energy consumption in the other hand. Our approach uses the clustering concept to divide the geographical area under subareas (clusters) of $R_{s}$ diameter to ensure full monitoring by a single node that can anyone receive in each cluster. The selected node is used as the Cluster-Head; i.e. supervise of the whole cluster node and to communicate the information to the base station. Communications within a cluster can be one-jump or a multi-jump based on backbone. The backbone could contain only cluster-heads or may include some gateway nodes to enable connectivity [6]. Calculating the connected dominating set is used for controlling the uncovered portions of areas and measuring the connectivity ratio in the network.

This paper presents a new approach based the benefits of using the Voronoï Diagram, Clustering and Connected Dominating Set in a hybrid model to achieve self-optimization for area coverage.

The remainder of the paper is organized as follows. In the next section (Section II), we describe related work on coverage optimization strategies. Section III presents firstly the general assumptions, and secondly provides the description of the proposed model. Section IV presents the evaluation results of our approach, and we finish by conclusion in Section V.

\section{RELATED WORK}

There are several ways to define coverage in WSNs, each has its advantages and its disadvantages. This section discusses few existing methods, and presents other known applications of Delaunay Triangulation (DT) and Vorono: Diagram (VD) in WSN. The Coverage Calculation Methods: which consist of the division of the area of interest into a grid of small squares, each representing one sensible area that should contain at least one sensor, as used by authors in [1], [7] is the simplest method for assuring sensing coverage on Area of Interest (AoI). The Delaunay Triangulation and Voronoï diagram are used to quantify the Quality of Coverage (QoC) in the empty spaces between sensors. They require a spatial segmentation algorithm whose characteristics reveal the QoC information. Among the possible techniques are the Voronoï algorithm, the Gabriel graph [8] and triangulation methods. Voronoï creates a polygon around each sensor. The Gabriel graph is a sub-graph of the Delaunay triangulation edge graph, so its edges divide the plane into larger polygons. A triangulation algorithm creates a graph of edges between sensors, which segments the plane into triangles, where many mathematical procedures could be used easier than on polygons with different numbers of vertices. Recently, Voronoï diagram was applied into coverage control for wireless sensor networks[5]. The AoI can be divided into several Voronoï cells. Each cell contains one sensor node. Every node could cover the cell using its circler monitoring radius. The circles in AoI are the sensing range of these nodes. Obviously, the coverage ratio is quite low and there exist holes in this case. The relationship between the sensing range of a node and its corresponding Voronoï cell may vary among the following cases: (a) the sensing range covers the Voronoï cell, (b) the Voronoï cell covers the sensing range, and (c) the sensing range and the Voronoï cell overlapped.

There are already lots of works in the literature focusing on the topic of area coverage and area coverage control in WSNs in order to improve the quality of service. Many researchers have been concerned by: area 1-coverage, area k-coverage, discovery and repairing the area k-coverage holes. Author G. Yaghoubi in [13] discussed the types of coverage problem according to different standards. He identified the coverage problem during the network deployment and provided an early solution while generating the network. Author in [2] defined a study to identify various problems related to coverage in sensor network. He provided a solution to cover the network area with a generation group over the network. He divided the network into smaller zones and then performed the coverage analysis. He also proposed a study on geometric computationbased, grid-based, etc. He studied these approaches under cost and benefit estimation. Author of [12] has defined the cover set generation based on disjoint set analysis. This kind of analysis is based on the cover set generation under disjoint set specification. He also defined an effective scheduling mechanism to set the order of cover set activation. The static environment and deterministic deployment are used since the location of each sensor can be predetermined properly. $M A X_{-} A V G_{-} C O V$ and $M A X_{-} M I N_{-} C O V$ are two gridbased algorithms proposed in [4], where sensors must be placed on predefined grid points distributed on the whole sensing area. These algorithms focus on average coverage as well as on maximizing the coverage of the most vulnerable grid points. $M A X_{-} A V G_{-} C O V$ tries to place sensors such that the average coverage of grid points will be maximized. In $M A X_{-} M I N \_C O V$, the coverage of grid point, that is less covered, will be maximized. In [10], authors consider an unreliable wireless sensor grid-network with nodes placed in a square of unit area. They derived sufficient and necessary conditions for the relations between coverage, connectivity and diameter. In [14], the sensing area was presented as an arbitrary-shaped polygon possibly with obstacles. The sensing area is partitioned into smaller sub-regions based on its shape, and then sensors are deployed into these regions systematically.

\section{THE DESCRIPTION AND THE IMPLEMENTATION OF THE HYBRID MODEL}

Our objective behind this hybrid model (Clustering, Voronoï Diagram, and Minimum Connected Dominating Set) is to benefit from the advantages and minimize the disadvantages of these three techniques in order to guarantee the coverage in WSN area. To apply our model, it is necessary to take some assumptions into consideration, such as: 
- Each node has a Num_Cluster parameter to designate the working set (group), which will be considered as a cluster in our strategy.

- We consider that each set of nodes occupying the same geographical subarea constructs a cluster of nodes that shares the same task, and each sensor node can be a Cluster-Head.

- Each node has one sensing range $\left(R_{s}\right)$ and two communication ranges; it communicates the collected data using the short-range communication $R_{c}^{s}$ to all neighboring nodes in the cluster, and it will transmit the data to the base station using the wide-range communication $R_{c}^{l}$, as described in the Fig. 1.

- The deployment of nodes is assumed to be dense and well dispersed on the area of interest.

- The base station is located in the center of the area of interest in a way that each node can communicate information directly to base station using the wide-range communication $R_{c}^{l}$.

This model consists of two selections, one at the level of each cluster (Sub-area) to choose the Cluster-Head and an another selection to construct the Voronoï diagram. The purpose of this diagram is to balance the new clusters (Voronoi Cells's) in the case where some sub-areas contain only exhausted nodes, that is to say the nodes of a cluster $k$ which are all depleted. In this case, the voronoï cells would be considered as new clusters. The selection of Clusters-Head in the new clusters (cells of voronö̈) and which will play the role of cover is as follows:

After the nodes' deployment step on the AoI; all nodes send a Hello message to the base station using the widecommunication range $R_{c}^{l}$. The $B S$ divides the AoI in sub-area zones and determines for each node their cluster and sends its their cluster numbers (Construction of the Clusters).

After each time period $T$, all Cluster $i$ nodes calculate their standardized energy reserve $\left(E_{N} o r^{i}\right)$ and send a Hello message containing $E_{N}$ or ${ }^{i}$ measurement for the (Selection of Clusters - Head).

The base station constructs the Voronoï Diagram in the AoI and sends all geographic positions at all activate nodes on the AoI. If a voronoï diagram cell contains more than one node, then the node which has the large energetic reserve will be activated as cluster-head and will realize the coverage in the entire polygon (the entire voronoï diagram cell). This model consists of one step to select the clusters. Another step is to select one Cluster-Head in each cluster which will play the role of covering the entire cluster and communicate the collected information towards the BS. The third step consists of calculating the Connected Dominating Sets (CDSs). This step is necessary to control the connectivity within each cluster and to measure the ratio of uncovered portions $\left(\rho_{j}\right)$ of each Cluster $j$. The fourth step is the construction of the Voronoi diagram. The purpose of this chart is to match the new clusters (Voronoï Cells) where some sub-areas contain only depleted nodes, that is to say that the nodes of a cluster $k$ are all depleted. In this case, the voronoi cells would be considered as new clusters. Selected Cluster-Head in new clusters (Voronoï cells), has to play the same role than the precedent ClusterHead.

the detailed description is as follows:

\section{Step 1 : Construction of the Clusters}

After their initial deployment, each sensor node sends a Hello message that contains the position to the base station. Based on this information, the base station divides the geographical area of dimension $D$ into sub-areas of dimension $L$, such as:

$$
L=\sqrt{2} \times R_{s}
$$

The base station repetitively sends just two points among the four corners of the square: the Cluster Number Num for each subarea and $P_{1}$ and $P_{4}$ or $P_{3}$ and $P_{4}$, and deduct the others two points. Interested nodes execute the algorithm 1 to see if it belongs to this subarea. If so, then the nodes will update their Cluster number. The pseudo algorithm showed in Algorithm 1 illustrates how to know if a point $P(x, y)$ is in the rectangle or not.

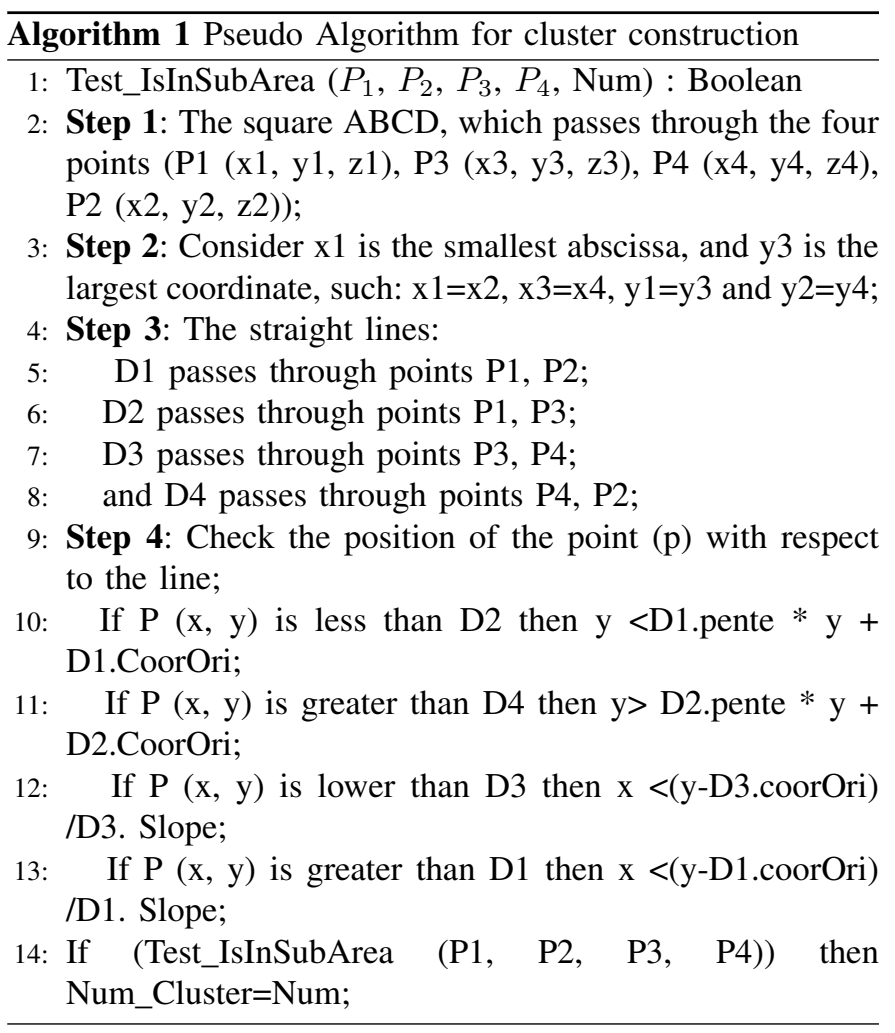

The subdivision of the geographic area by $R_{s}$ diameter of subareas aims to ensure the whole monitoring of any node in the cluster, and to ensure connectivity (communication between nodes), as shown in Fig. 5 and Fig. 2.

Step 2 : Selection of Clusters - Head

We consider each subarea as a cluster and each sensor node can be a Cluster-Head. The neighbor node that has the largest 
energy reserve elected as a Cluster-Head $(\mathrm{CH})$. The selection of $C H$ works as follows:

The nodes $i$ of each cluster $j$ calculate their standard energy reserves $\left(E_{N o r}^{i j}\right)$.

$$
E_{\text {Nor }}^{i j}=\frac{E_{\text {Act }}^{i j}}{E_{0}}
$$

Where:

$E_{A c t}^{i j}$ : Represents the energy reserve remained of the node (i.e. $n_{i j}$; the node $i$ of the cluster $j$ ).

$E_{0}$ : represents the initial energy of a sensor node.

The $\mathrm{CH}$ is elected based on a highest metric that combines the Euclidian distance to the center and the remaining energy of the node.

The $\mathrm{CH}$ node communicates the data collected using the short-range communication $R_{c}^{s}$ to to elected neighbor node (Cluster-Head). This node will transmit the data to the base station using the wide-range communication $R_{c}^{l}$ to minimize the energy consumption and keep the perfect coverage of the area of interest.

Step 3 : Calculating the Connected Dominating Sets

This step is necessary to control the connectivity within each cluster and to measure the ratio of uncovered portions $\left(\rho_{j}\right)$ of each Cluster $j$, i.e. see if there is in each cluster at least one Minimal Connected Dominating Set $(M C D S)$. Therefore, if $\rho_{j}=1$, then, the Cluster $j$ is uncovered; if $\left.\rho_{j} \in\right] 0 . .1[$, then, the cluster is partially uncovered; otherwise $\left(\rho_{j}=0\right)$, the cluster is fully covered.

\section{Step 4 : Construction of Voronoi Diagram}

This step is necessary if there are some empty clusters or the ratio of uncovered portions clusters is not null $\rho_{j} \neq 0$. The $B S$ constructs the $A o I$ Voronoï Diagram to delineate the new clusters. After that, it returns to each active node (probable Clusters-Head) on $A o I$ all the positions and thus their cluster numbers that they contain. This aims to reach the full coverage on the whole interest area with the rest of the living sensor nodes in the network. Otherwise, the base station keeps the same old cluster structure. After a predetermined period $T$ by the base station; all nodes will be activated and will they send a Hello message to the neighboring nodes in the cluster containing the information of the energy left using short-communication range $\left(R_{c}^{s}\right)$. The current $C H$ sends more gathered information during its monitoring period using $R_{c}^{s}$ to neighboring nodes. The nodes in each cluster compare their left energy with other neighbors. Then, the node that has the largest energy reserve will automatically be activated and be considered as the cluster-head. However, the other nodes will return to the passive state. New $\mathrm{CHs}$ send Hello messages to the base station (containing the geographical position and the collected information in the previous period) using the $R_{c}^{l}$. The $B S$ calculates the ratio of the uncovered portion $(\rho)$ and decides if it is necessary to construct the clusters by Voronoï

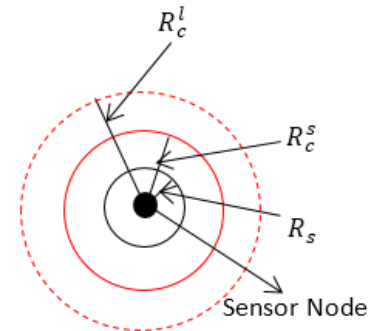

Fig. 1. Communication and Sensing ranges.

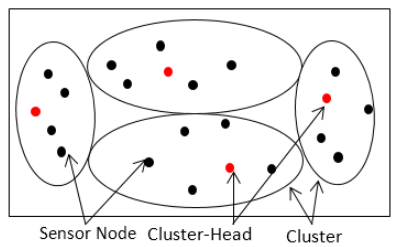

Fig. 2. Clustering Concept.

Diagram.

These steps will run until the exhaustion of the whole network. Fig. 2, shows an example of the subdivision of the geographic area, Clusters, $C D S s$ and Clusters-Head.

\section{Simulation and Evaluation}

In this step, we have defined the protocols used in the simulation, cited their strengths and weaknesses in order to make a good comparison. Then, we quoted the parameters of the simulation, and presented the simulator used. We ended this part by analyzing the results found in terms of the

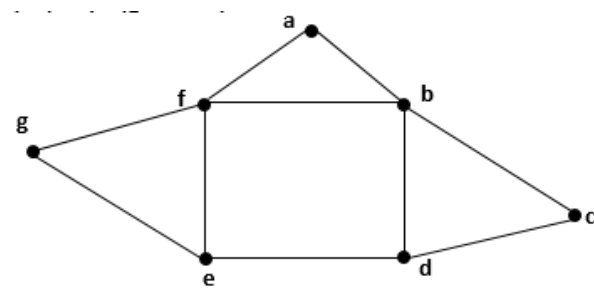

Fig. 3. $\{g, b\},\{g, a, c\}$ and $\{g, a, c, d\}$ are CDSs, $\{f, d\}$ is Minimum Connected Dominating Set.

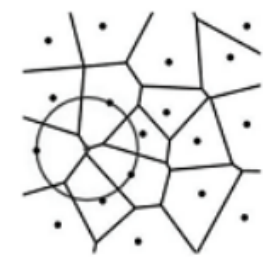

Fig. 4. Vorono $\tilde{A}^{-}$Diagram.[3] 


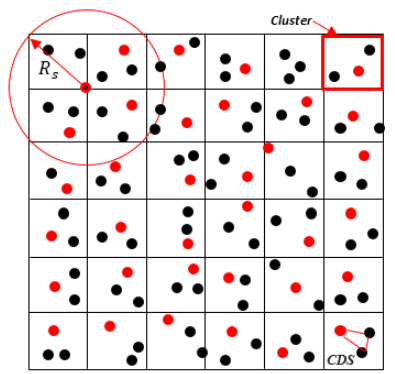

Fig. 5. Subdivision of AoI; the Clustering, CDSs and CHs.

coverage rate, the rate of energy consumption consumed and in terms of the rate of nodes remaining alive.

\section{A. Strengths and weaknesses of simulation protocols}

- The NLAC protocol uses Clustering as a node activation planning technique (active / passive), but the one-time clustering application to allow for the proper distribution of the sensor nodes does not show a major benefit in the coverage. other planning mechanisms are needed to allow the network to be redistributed equitably.

- The IDA-OFCA protocol is proposed to make a fully distributed decision to select the active nodes, but to ensure the good distribution of the sensor nodes in the network by the mechanism used by IDA-OFCA it is necessary that the network is very dense and well deployed., the more it requires the mobility of sensor nodes as a condition to keep the distribution fair in the network. The thing that quickly depletes the network.

- The VAC protocol is proposed to address weaknesses of protocols that rely on completion to equitable distribution. This protocol uses clustering, and Voronoï diagram to ensure maximum coverage, based on decision algorithms that use greedy calculation of the distribution, the thing that exhausts the network in the first rounds of application of this protocol.

\section{B. Simulations Parameters}

The experiments were conducted using MATLAB Simulator. All the sensor nodes are homogeneous(same widecommunication range, short-communication range and the same sensing range). The simulation parameters used are listed in Table I [5].

\section{Simulation illustration}

The base station divides the area of interest using $R_{s}=28.28 \mathrm{~m}$ as diameter of each square cell (cluster). With a well dispersed deployment of 200 sensor nodes, each cluster can contain from 5 to 6 sensor nodes. The duration of the simulation is $1000 \mathrm{~s}$, which contains 50 Rounds. We scattered different five (5) trials (varying from 200, 400, 600, 800 and 1000 of sensor nodes) in the AoI with uniform density. We evaluated the average of the coverage ratio and the average of the energy consumption while varying the number of deployed
TABLE I

SIMULATION PARAMETERS

\begin{tabular}{|l|c|}
\hline Parameters & Value \\
\hline Shape of the monitored area & Square \\
\hline Size of the monitored area & $170 \mathrm{~m} X 170 \mathrm{~m}$ \\
\hline Number of sensor nodes & $200,400,600,800,1000$ \\
\hline Wide-Communication range & $131.24 \mathrm{~m}$ \\
Short-Communication range & $56.56 \mathrm{~m}$ \\
\hline Sensing range & $28.28 \mathrm{~m}$ \\
\hline Initial energy & $100 \mathrm{~J}$ \\
\hline Data transfer ratio & $250 \mathrm{kbps}$ \\
Time total of simulation & 1000 seconds \\
\hline Round Time & $20 \mathrm{~S}$ \\
\hline Rounds Number & 50 \\
\hline
\end{tabular}

sensor nodes on the AoI. We compare our proposed protocol denoted $V D \_C \_C D S$ with three (3) state of art works, namely $N L A C$ [2], IDA-OFCA [12] and $V A C$ [11]. The simulation results of the average coverage percentage according to the number of deployed nodes after 50 rounds are shown in Fig. 6.(a)

The simulation results of average energy consumption percentage of monitoring according to the number of deployed nodes after 50 rounds are shown in Fig. 6.(b)

Simulation results showing the number of sensor nodes percentage remain active after 50 rounds are presented in Fig. 6.(c)

\section{Results Analysis}

1) The Coverage Rate: Fig. 6.(a) shows the average percentage of AoI coverage of the four (4) tested protocols after 50 times of $B S$, according to number of deployed nodes within the 5 trials over the AoI. Our proposed protocol ( $V D \_C \_C D S$ ) shows a full coverage progress of the AoI from $94 \%$ to $96.5 \%$ depending on the number of sensor nodes, which is fairly scattered on $A o I$, and for different densities of deployed networks, especially, when the density is dense knots of the long coverage lasts through Voronoï-based area coverage mechanism $(V A C)$ protocol against the protocols: $N L A C$ and $I D A \_O F C A$ show less coverage and they do not exceed $95 \%, 90 \%$ and $77.5 \%$ respectively for the $V A C$, $N L A C$ and IDA_OFCA after 50 rounds, when the nodes density reaches 1000 nodes.

2) Energy Consumption Rate: Fig. 6.(b) shows the average energy consumption to ensure coverage of the four protocols after 50 times of $B S$ according to the number of deployed nodes within 5 trials. Our proposed protocol $\left(V D \_C \_C D S\right)$ shows a minimal and efficient energy consumption to ensure full area coverage by deployment of the sensor nodes, from $65 \%$ for 200 nodes to $15.5 \%$ for 1000 deployed nodes of the network total energy after 50 rounds. However, the three others protocols show energy consumption reduction at $18 \%$, $32 \%$ and $58.5 \%$ respectively for the $V A C, N L A C$ and IDA_OFCA even when the density of nodes arrived to 1000 nodes. 


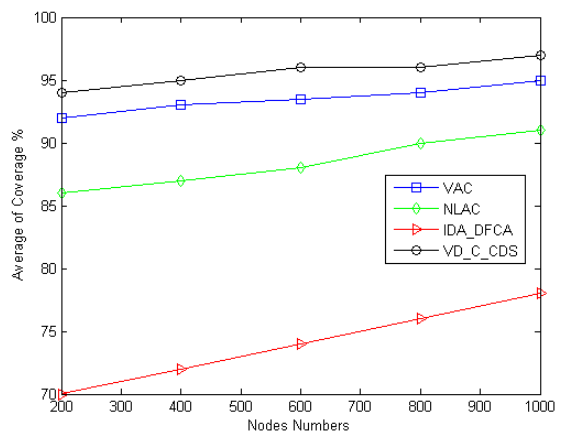

(a)

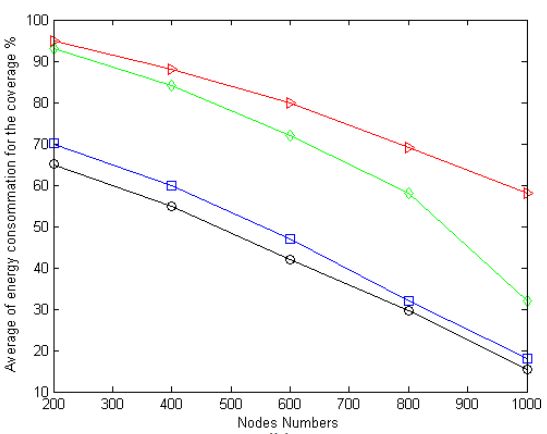

(b)

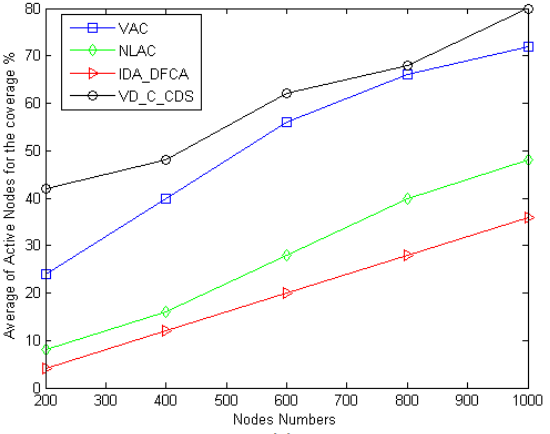

(c)

Fig. 6. (a.) The average coverage percentage VS the number of deployed nodes. (b.) The average energy consumption of monitoring percentage VS the number of deployed nodes. (c.) the number of sensor nodes percentage remaining active VS the number of deployed nodes.

3) The Remained Active Rate: The Fig. 6.(c) shows the rate of remained active nodes of the four protocols after 50 times of $B S$ a final according to the number of deployed nodes of 5 trials. Our protocol shows its effectiveness through the large number of remained sensor nodes. In fact, it reached a percentage of $80 \%$ at a density of 800 nodes after 50 rounds times. The $V A C, N L A C$ and IDA_OFCA show a percentage reached to $70 \%, 48 \%, 40 \%$ for 1000 sensor network nodes.

\section{CONCLUSION}

The three techniques; (a) Voronoï Diagram, (b) Minimum Connected Dominating Set, and (c) Clustering are among the best optimization techniques already proposed in the area coverage in WSNs. Our contribution is to hybridize these techniques to gain the benefits and minimize the disadvantages of these techniques. A technical hybrid Model of: Clustering, Connected Dominating Sets and Voronoï Diagram is implemented to optimize a NP-Hard area coverage problem. The main idea behind the use of clustering was to activate a single sensor node in each cluster; for playing a multiple role; a role of Cluster-Head, a role of coverage in the entire cluster and a role of communication sender of the information to the base station.

To check the connectivity in each cluster and to measure the portion rate of uncovered areas, we used the $C D S s$ concept. If the rate of uncovered portions is not null, then the Voronoï Diagram reconstruction is required. Thanks to benefits of the proposed protocol, the application reach a coverage of $96.5 \%$ of AoI, as shown in the simulation results, and preserve the connectivity until the network depletion. As a future works, we intend to use the proposed hybrid technique to apply it into 3D WSN coverage study. We aim to study its efficiency in $3 \mathrm{D}$ cases.

\section{REFERENCES}

[1] S. Abd. Razak, H. Chizari, A. Bahar, and A. H. Abdullah. Deployment density estimation for a-covering problem in wireless sensor network. In International Symposium on Information Technology, page 592-596, Kuala Lumpur, Malaysia, June, 2010.
[2] N. Aslam and W. Robertson. Distributed coverage and connectivity in three dimensional wireless sensor networks. In IWCMC 10 Proceedings of the 6th International Wireless Communications and Mobile Computing Conference ACM, pages 1141-1145, Caen, France, June 28- July 2. 2010.

[3] K.-C. L. Chun-Hsien Wu and Y.-C. Chung. A delaunay triangulation based method for wireless sensor network deployment. In Proceedings of the 12th International Conference on Parallel and Distributed Systems (ICPADS 06). IEEE, 2006.

[4] S. S. Dhillon and K. Chakrabarty. Sensor placement for effective coverage and surveillance in distributed sensor networks. In Wireless Communications and Networking, 2003. WCNC 2003. 2003 IEEE, volume 3, pages 1609-1614. IEEE, 2003.

[5] L. C. B. Z. Guoyong Dai, Hexin Lv and P. Xu. A novel coverage holes discovery algorithm based on voronoi diagram in wireless sensor networks. International Journal of Hybrid Information Technology,9(3):273$282,2016$.

[6] A. N. Hai Liu and I. Stojmenovic. Wireless Sensor and Actuator Networks, chapter Chap 1.14: problems at the Network layer, pages 19-50. John Wiley and Sons, Inc., Hoboken, New Jersey, 2010.

[7] H. W. Haining Chen and N.-F. Tzeng. Grid-based approach for working node selection in wireless sensor networks. In 2004 IEEE International Conference on Communications, volume 6, page 3673, University of Louisiana, Lafayette, LN, USA, June?S24 2004.

[8] N. J. Ijaz Muhammad Khan and S. Zeadally. Hop-based approach for holes and boundary detection in wireless sensor networks. IET Wireless Sensor Systems, 2(4):328-337, 2012.

[9] J. M. H. . J. L. H. . M. J. Mossinghoff. Combinatorics and Graph Theory, chapter Undergraduate Texts in Mathematics. Springer, NY, New York, NY, USA, second edition, 2008.

[10] N. S. S. Shakkottai, R. Srikant. Unreliable sensor grids: Coverage, connectivity and diameter. In IEEE INFOCOM, pages 1073-1083. IEEE, 2003.

[11] M. A. R. Selina Sharmin, Fernaz Narin Nur and M. M. Rahman. Area coverage for clustered directional sensor networks using voronoi diagram. In IEEE International WIE Conference on Electrical and Computer Engineering (WIECON-ECE), BUET, Dhaka, Bangladesh, 19-20 December 2015.

[12] Y. S. C. Vijay Chandrasekhar, Winston KG Seah and H. V. E e. Localization in underwater sensor networks : survey and challenges. In WUWNet '06 Proceedings of the 1st ACM international workshop on Underwater networks, number 1-59593-484-7/06/0009, pages 33-40. ACM, Los Angeles, California, USA, September 252006.

[13] G. Yaghoubi. Connectivity issue in wireless sensor networks by using depth-first search and genetic algorithm. In Computational Intelligence and Communication Networks (CICN), 2010 International Conference on Computational Intelligence and Communication Systems, pages 377-381, Bhopal, 26-28 November 2010.

[14] C. H. Y.C. Wang and Y. Tseng. Efficient deployment algorithms for ensuring coverage and connectivity of wireless sensor networks. In Wireless Internet Conf. (WICON, 2005.

[15] M. Rigo. Theorie des graphes, pages 1-176, Deuxiemes bacheliers en sciences mathematiques Annee academique, Faculte des sciences Departement de mathematiques, 2009-2010. 